\title{
Dipole-quadrupole interactions and the nature of phase III of compressed hydrogen
}

\author{
Jorge Kohanoff ${ }^{1}$, Sandro Scandolo ${ }^{2,3}$, Stefano de Gironcoli ${ }^{2,3}$ and Erio Tosatti ${ }^{1,2,3}$ \\ 1) International Centre for Theoretical Physics, \\ Strada Costiera 11, I-34014 Trieste, Italy \\ 2) International School for Advanced Studies (SISSA) \\ via Beirut 4, I-34014 Trieste, Italy \\ 3) Istituto Nazionale Fisica della Materia (INFM)
}

\begin{abstract}
A new class of strongly infrared (IR) active structures is identified for phase III of compressed molecular $\mathrm{H}_{2}$ by constant-pressure ab initio molecular dynamics and density-functional perturbation calculations. These are planar quadrupolar structures obtained as a distortion of low-pressure quadrupolar phases, after they become unstable at about $150 \mathrm{GPa}$ due to a zone-boundary soft phonon. The nature of the II-III transition and the origin of the IR activity are rationalized by means of simple electrostatics, as the onset of a stabilizing dipole-quadrupole interaction.
\end{abstract}

The nature of the high-pressure phases of solid hydrogen is of long time interest [1]. One of the most intriguing and outstanding issues is the nature of the 150 GPa phase transition [2 and the origin of the strong infrared vibronic activity observed in the high pressure phase [3]. The present view of the low temperature phase diagram of hydrogen is the following. In the gas and diluted phases the homopolar $\mathrm{H}_{2}$ molecules interact via electric quadrupole-quadrupole interactions (EQQ). At low temperatures, quantum rotational effects overcome librational barriers, so that the molecules rotate freely. Upon compression, EQQ interactions freeze the molecular rotations into an ordered broken-symmetry phase (BSP, or phase II). Above $\sim 150 \mathrm{GPa}$, a third phase (H-A, or phase III) is observed. Optical measurements across the II-III phase boundary indicate a substantial increase in the vibron infrared (IR) activity, the effective charge being one order of magnitude larger in phase III than in phase II [4]. The lack of a Drude edge in extended IR studies down to $1000 \mathrm{~cm}^{-1}$ indicates that phase III is still insulating [5]. More recent optical experiments focused on the low-frequency region of the vibrational spectrum [6], revealed the sudden appearance in phase III of at least twelve modes below $1000 \mathrm{~cm}^{-1}$, whose frequencies strongly increase with pressure, extrapolating to zero at $P \leq 150 \mathrm{GPa}$, and suggesting that quantum librational effects are frozen out and are not of paramount importance in phase III [7].

A satisfactory theory of the origin of the IR activity and the nature of the II-III phase transition is still unavailable. First-principles calculations, although numerous, have failed so far to provide a unique description of phase III 8 13, even after inclusion of proton quantum effects 14 16. Some of them failed in properly sampling the electronic Brillouin zone [10,15, 16, others in considering unit cells too small to be consistent with the multiplicity of low frequency modes [8,9,11 14. In particular, some structures studied were metallic [12], and the $C m c 2_{1}$ structure, which is currently being considered as a promising candidate for phase III [9, 13, 17], turns out to be unstable against $P c a 2_{1}$, at least within LDA and
GGA.

In this letter we report the results of a combined structural and vibrational study using constant-pressure ab initio molecular dynamics simulations (CPAIMD) 18 and density functional perturbation theory (DFPT) calculations [19]. These show that, starting with the likeliest structure proposed for phase II, a zone-boundary phonon instability sets in at about $150 \mathrm{GPa}$. The resulting stable structures have larger unit cells and exhibit enhanced IR activity, precisely the features typical of phase III. We then show that the transition from low to high IR activity can be understood in terms of simple electrostatics, as a structural transition driven by dipole-quadrupole interactions.

The details of the ab initio calculations are the following. Electronic correlations were treated within a gradient corrected density functional [20], while the protonelectron interaction was described through a local pseudopotential 21] that requires an energy cutoff of $60 \mathrm{Ry}$ for the plane wave expansion. Convergence on BZ sums required $512 k$-points in the full BZ [22] of 4 and 6molecule supercells, and $256 k$-points in an 8-molecule supercell. In the MD simulations, full BZ sampling was implemented using a self-consistent $k \cdot p$ technique 12,23,24.

Previous CPAIMD simulations indicate that the quadrupolar $P c a 2_{1}$, hcp structure (see Fig. 1) is likely to correspond to the low-pressure phase II [23], confirming that the EQQ are dominant at low densities. In the present work we have computed the zone-center and zone-boundary ab initio phonon frequencies at $140 \mathrm{GPa}$ using DFPT, and found that this structure is, in fact, stable. However, increasing pressure to $180 \mathrm{GPa}$, the phonon dispersion of the $P c a 2_{1}$ structure displays an instability at the (100) Brillouin zone boundary [25]. Using the corresponding eigenvector we distorted the original structure and minimized the enthalpy by optimizing lattice parameters and molecular orientations. In the 8molecule structure derived from the $P c a 2_{1}$, and shown in Fig. 1, we found a tendency of the molecules to lie in parallel, (001) hcp planes. This tendency becomes more pronounced the higher the pressure. 
Once molecules lie in, or close to planes, it is natural to surmise that another possible candidate would be a structure that optimizes the quadrupolar interactions in a triangular, two-dimensional system. Within the planes, neighboring molecules are oriented at 60 degrees from each other. This minimizes frustration of EQQ interactions, an angle of 90 degrees being impossible in a triangular lattice. Thus, we also optimized the structure of the triangular structure by means of CPAIMD, in a 6-molecule cell (see Fig. 1), and found it to be stable, with an enthalpy lower than that of the distorted $\mathrm{Pca} 2_{1}$. In this way we obtained a new class of structures which are characterized by unit cells containing more than 4 molecules.

In order to make a direct comparison with experimental data, we report in Fig. 2 the vibrational spectrum at zone center of the undistorted $P c a 2_{1}$ structure at 140 $\mathrm{GPa}$ and, at higher pressures, where this structure becomes unstable, the spectrum of the centrosymmetric triangular structure. We first notice that the discontinuous frequency drop upon transformation from the $P c a 2_{1}$ to the triangular structure, compares favorably with an experimental jump of about $100 \mathrm{~cm}^{-1}$. This jump is connected with an intramolecular bond softenining signalled by a sudden, $\approx 2 \%$ increase in the molecular bond lengths. Actually, in the triangular structure, the bond length is 1.41 Afor two of the six molecules in the unit cell, and 1.39 Afor the other four. In the Pca2 structure the bond length is $1.37 \AA$. Concomitantly, we find $\mathrm{a} \approx 1 \%$ discontinuous volume decrease at the transition. In fact, the main energy decrease at the transition is in the interproton repulsion, counteracted by a nearly equal increase of electronic energy. The lower part of the experimental IR and Raman spectra of phase III shows a number of modes whose frequencies extrapolate to zero for decreasing pressure, between 100 and 150 $\mathrm{GPa}$, but are totally absent in phase II [6]. Since optics detects only zone-center modes, these data strongly suggest a zone-boundary libron instability of phase II, with formation of a larger supercell and soft mode refolding at $k=0$ in phase III. This is precisely what our first-principles calculations predict. The mode-hardening with pressure which we calculate for the lowest frequency mode is $41.5 \sqrt{(P-142)} \mathrm{cm}^{-1}$ (see Fig. 2).

The DFPT approach also allows us to compute IR activities [26], which we report in Table I, as a function of pressure. At low pressures three of the vibrons are nominally IR active, but the oscillator strengths are very small, with effective charges $Z^{*} \approx 0.02 e$. After the transition to the high-pressure phase, the IR activity increases suddenly by one order of magnitude, i.e. $Z^{*} \approx 0.2 e$. This large jump appears to account quantitatively for the experimental onset of strong IR activity in phase III [4]. The IR activity is further shown, using effective charges calculated at $220 \mathrm{GPa}$, to grow steadily by about $1.5 \times 10^{-4} \mathrm{e} / \mathrm{GPa}$ with increasing pres- sure, to be compared with an experimental increase of $2.07 \times 10^{-4} e / \mathrm{GPa}$. Finally, there is an IR phonon mode at about $1600 \mathrm{~cm}^{-1}$, which may explain that seen in one set of experiments [5].

An intriguing fact is that we find three IR active vibrons against only one observed experimentally. We note here that this threefold multiplicity is of strictly interplanar nature. Interplanar interactions cause, e.g. the splitting between modes A (IR active) and B (Raman active), as indicated in Fig. 2. If angular correlations between planes were to be reduced, or eliminated, for example by librational fluctuations, then the AB doublet would collapse, and the IR activity of mode A would be supressed. The alternative possibility of a smaller unit cell (3 molecules) is not supported by our calculations, and seems also incompatible with the large number of experimental librational modes.

We computed, in addition, the static dielectric constant, which is experimentally accessible via refractive index measurements [27]. We predict a discontinuous increase of about $10 \%$ at the $150 \mathrm{GPa}$ phase transition, which is at variance with the decrease calculated for other simpler structures on the basis of a dielectric catastrophe mechanism, driven by diverging dipole-dipole interactions [9].

We now address the physics underlying the II to III transition, which appears to be driven by dipolequadrupole interactions. In fact, a simple electrostatic model consisting of interacting quadrupoles and corresponding induced dipoles in a hcp lattice qualitatively accounts for the picture emerging from the above firstprinciples results. This model is an extension of the original model of interacting pure quadrupoles 28] with the addition that the $\mathrm{H}_{2}$ molecules are polarizable, and develop a dipole moment when placed in an external field such as that resulting from all the quadrupoles of the neighboring molecules. Once these induced dipole moments set in, dipole-quadrupole (EDQ) and dipole-dipole (EDD) interactions add to the basic EQQ interactions. These depend differently on the intermolecular distance $\left(1 / r^{5}\right.$ for EQQ, $1 / r^{4}$ for EDQ, and $1 / r^{3}$ for EDD), thus originating a competition between different orientational patterns 29].

In Fig. 3 we show the energy diagram and the behaviour of the dipole moment as a function of the lattice constant. When the molecules are far apart the EQQ interaction is dominant and the Miyagi-Nakamura result is recovered [28]: in the hcp structure for the molecular centers, a $P c a 2_{1}$ structure is favored, the molecules arranging themselves as close to perpendicular as they can. As the distance between molecular centers decreases, all individual quadrupolar electric fields on a given molecule increase, inducing a molecular dipole moment. The EDQ interaction, is however still weak in $P c a 2_{1}$ because in this structure the quadrupolar fields due to neighboring molecules almost cancel, and the main contribution to 
the energy remains EQQ. With decreasing spacing, the increasing dipole moment would eventually drive a dielectric instability [9], as the electric fields created by the induced dipoles interact cooperatively. As any two molecules approach under pressure, however, the increasing EDQ interaction causes their relative orientation angle to turn, driving a $k \neq 0$ libron instability and eventually stabilizing a new planar triangular structure. The dielectric instability is therefore preempted by a structural transition. Remarkably, the structure so obtained is very close to the planar quadrupolar structure with in-plane quadrupolar orientations just surmised from ab initio calculations [31].

The quadrupolar electric field at each molecular centre is now between 5 and 15 times larger than in the $P c a 2_{1}$ structure due to incomplete cancellation of the contributions of the neighboring molecules. This field induces proportionally larger dipole moments, so that the EDQ interaction turns out to be more than two orders of magnitude larger, and comparable in strength to the EQQ interaction. At the transition distance, the static molecular dipole moment jumps by a factor of about 8 , closely paralleling that of the dynamical dipole calculated by DFPT, which is related to the IR activity. The jump of IR activity thus signals a very central aspect of the physics driving the II-III transition.

In conclusion, we have presented a theory that sheds light on the nature of phase III of solid molecular hydrogen, and explains the origin of its strong IR activity. The quadrupolar electric field of the $\mathrm{H}_{2}$ molecules induces a dipole moment in the polarizable neighboring molecules. The ensuing dipole-quadrupole interaction, gives rise to a nonuniform librational instability driving a phase transition to a planar structure, still exhibiting inplane quadrupolar ordering, but also optimizing the new term. The corresponding candidate structures obtained from first-principles calculations possess larger unit cells, and reproduce many measured properties, including the vibronic effective charges, the vibron discontinuities, the librational phonon spectrum, and the insulating character of this phase. The exact symmetry group of phase III and the precise reason for a single IR mode remain open questions, calling for further theoretical and experimental work.

[1] E. Wigner and H. B. Huntington, J. Chem. Phys. 3, 764 (1935); H. K. Mao and R. J. Hemley, Rev. Mod. Phys. 66, 671 (1994) and references therein.

[2] R. J. Hemley, and H.-K. Mao, Phys. Rev. Lett. 61, 857 (1988); H. E. Lorenzana, I. F. Silvera, and K. A. Goettel, Phys. Rev. Lett. 63, 2080 (1989).

[3] M. Hanfland, R. J. Hemley, and H.-K. Mao, Phys. Rev.
Lett. 70, 3760 (1993).

[4] R. J. Hemley, I. I. Mazin, A. F. Goncharov, and H.-K. Mao, Europhys. Lett. 37, 403 (1997).

[5] N. H. Chen, E. Sterer, and I. F. Silvera, Phys. Rev. Lett. 76, 1663 (1996); R. J. Hemley et al., Phys. Rev. Lett. 76, 1667 (1996).

[6] A. F. Goncharov, R. J. Hemley, H.-K. Mao, and J. F. Shu, Phys. Rev. Lett. 80, 101 (1998).

[7] I. I. Mazin, R. J. Hemley, A. F. Goncharov, M. Hanfland, and H.-K. Mao, Phys. Rev. Lett. 78, 1066 (1997).

[8] B. Edwards, N. W. Ashcroft, and T. Lenosky, Europhys. Lett. 34, 519 (1996).

[9] B. Edwards and N. W. Ashcroft, Nature 388, 352 (1997).

[10] J. S. Tse and D. D. Klug, Nature 378, 595 (1995).

[11] A. Alavi, Phil. Trans. R. Soc. Lond. A 356, 263 (1998).

[12] J. Kohanoff and S. Scandolo, Mat. Res. Soc. Proc. Symp. 499, 329 (1998).

[13] K. Nagao and H. Nagara, Phys. Rev. Lett. 80, 548 (1998).

[14] M. P. Surh, T. W. Barbee III, and C. Mailhiot, Phys. Rev. Lett 70, 4090 (1993).

[15] V. Natoli, R. M. Martin and D. M. Ceperley, Phys. Rev. Lett. 70, 1952 (1995).

[16] S. Biermann, D. Hohl, and D. Marx, J. Low Temp. Phys. 110, 97 (1998).

[17] I. Souza and R. M. Martin, Phys. Rev. Lett. 81, 4452 (1998).

[18] P. Focher et al., Europhys. Lett. 36, 345 (1994).

[19] S. Baroni, P. Giannozzi and A. Testa, Phys. Rev. Lett. 58, 1861 (1987).

[20] The LDA was implemented as in J. P. Perdew and A. Zunger, Phys. Rev. B 23, 5048 (1981), and gradient corrections as in A. D. Becke, Phys. Rev. A 38, 3098 (1988); J. P. Perdew and Y. Wang, Phys. Rev. B 33, 8822 (1986).

[21] P. Giannozzi (unpublished). See also F. Gygi, Phys. Rev B 48, 11692 (1993).

[22] H. J. Monkhorst and J. D. Pack, Phys. Rev. B 13, 5188 (1976).

[23] J. Kohanoff, S. Scandolo, G. L. Chiarotti and E. Tosatti, Phys. Rev. Lett. 78, 2783 (1997).

[24] S. Scandolo and J. Kohanoff (unpublished).

[25] The minimum of the phonon dispersion is not necessarily at zone boundary (cell doubling). The new stable cell could be larger, or even incommensurate. A 3/2 unit cell would correspond to the triangular lattice mentioned later in the text.

[26] IR activity is here defined in terms of effective charges, $Z^{*}=\mu f \Omega / 4 \pi$, where $\mu$ is the reduced mass of the molecule, $f$ the oscillator strength, and $\Omega$ the molecular volume.

[27] W. J. Evans and I. F. Silvera, Phys. Rev. B 57, 14105 (1998).

[28] H. Miyagi and T. Nakamura, Prog. Theor. Phys. 37, 641 (1967).

[29] In order to mimick the $\mathrm{H}_{2}$ molecule, we have used a cylindrically symmetric (traceless) quadrupolar tensor with a quadrupole moment of 0.5 a.u., and a polarizability tensor with a value of $6.7 \mathrm{a} . \mathrm{u}$. for the component parallel to the molecular axis, and 4.7 a.u. for the orthogonal component 30]. The model total energy includes EDD, EDQ, EQQ, and the electrostatic energy, and is minimized for 
variable structure and spacing. The molecular dipole moments are calculated by assuming a linear polarizability constitutive relationship.

[30] J. Van Krankendonk, Solid Hydrogen (Plenum, NY, 1983), pp. 15-19. We have taken the $c / a$ ratio of the hcp cell smaller than the ideal value, according to former ab initio results 23.

[31] The triangular structure obtained from the model differs from the ab initio one by a sliding of the triangular planes, while the in-plane stuctures are identical.

FIG. 1. Schematic view of the low-pressure quadrupolar phase $P c a 2_{1}$ (a), and two candidates for phase III: (b) the distorted $P c a 2_{1}$, and (c) the triangular structure. Large and small circles indicate that the molecule lies out of the plane, pointing in the direction of the larger circle. Empty and shaded molecules correspond to different planes.

FIG. 2. Zone-center phonon spectrum as a function of pressure for the $P c a 2_{1}$ structure at $140 \mathrm{GPa}$ (shifted to the left for the sake of clarity), and the triangular structure, above $140 \mathrm{GPa}$. The pressure dependence of the IR active vibrons is indicated with dashed lines, and that of the soft librational mode with a solid line, crossing zero at $P \approx 142 \mathrm{GPa}$.

FIG. 3. Model of quadrupoles and induced dipoles: Energy (left panel) and dipole moment (right panel) per molecule as a function of the lattice constant, in an hcp lattice. The dipole moment exhibits a discontinuity of nearly one order of magnitude at the transition distance.

TABLE I. Vibron effective charges as a function of pressure. Values are reported for the Pca $2_{1}$ structure at $140 \mathrm{GPa}$, and for the triangular structure at 180 and $220 \mathrm{GPa}$. Experimental values were taken from Ref. [4].

\begin{tabular}{ccc}
\hline \hline$P(\mathrm{GPa})$ & $\mathrm{Z}^{*}$ (calc.) & $\mathrm{Z}^{*}$ (exp.) \\
\hline 140 & 0.02 & 0.011 \\
180 & 0.19 & 0.10 \\
220 & 0.21 & 0.13 \\
\hline \hline
\end{tabular}




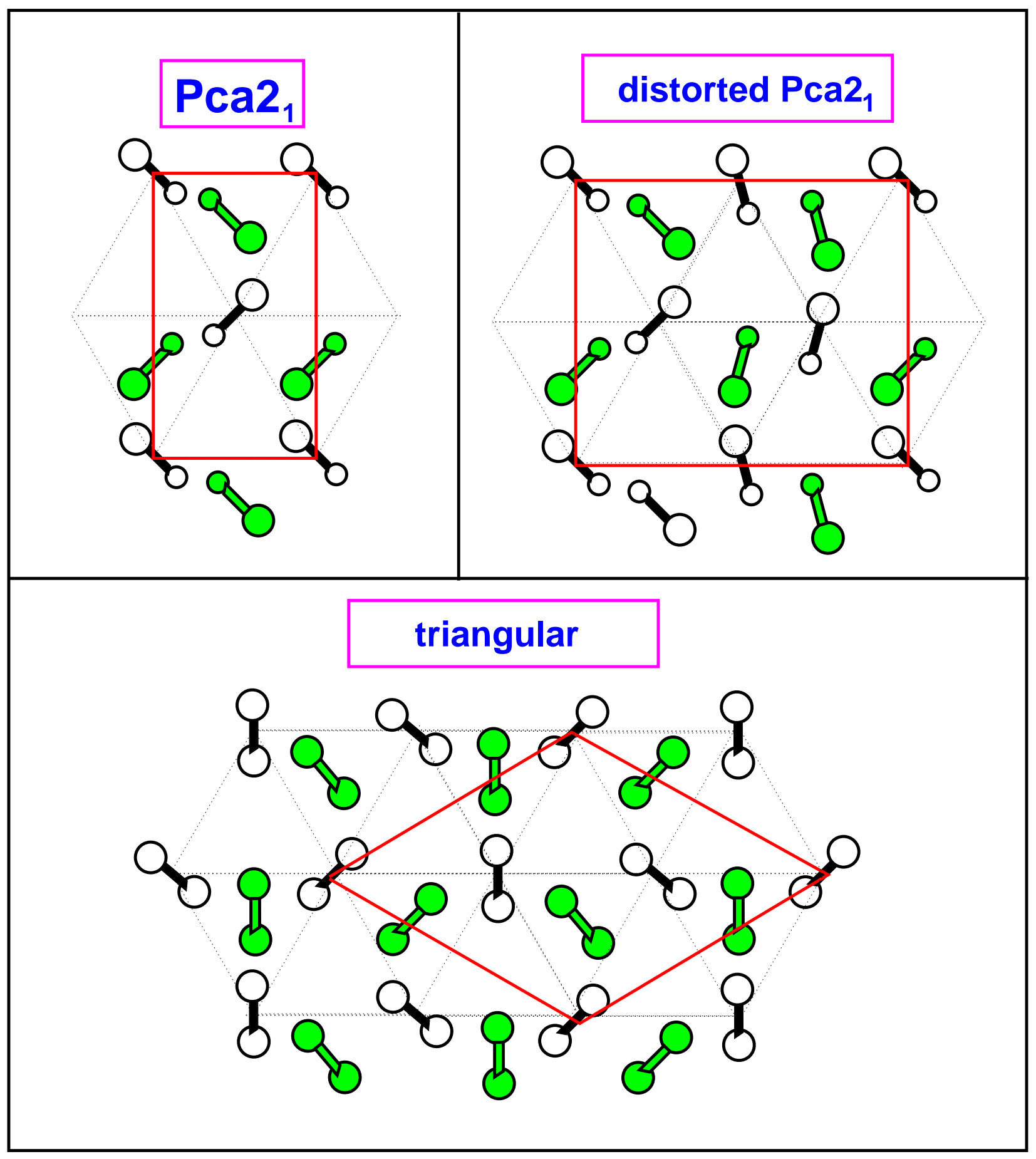




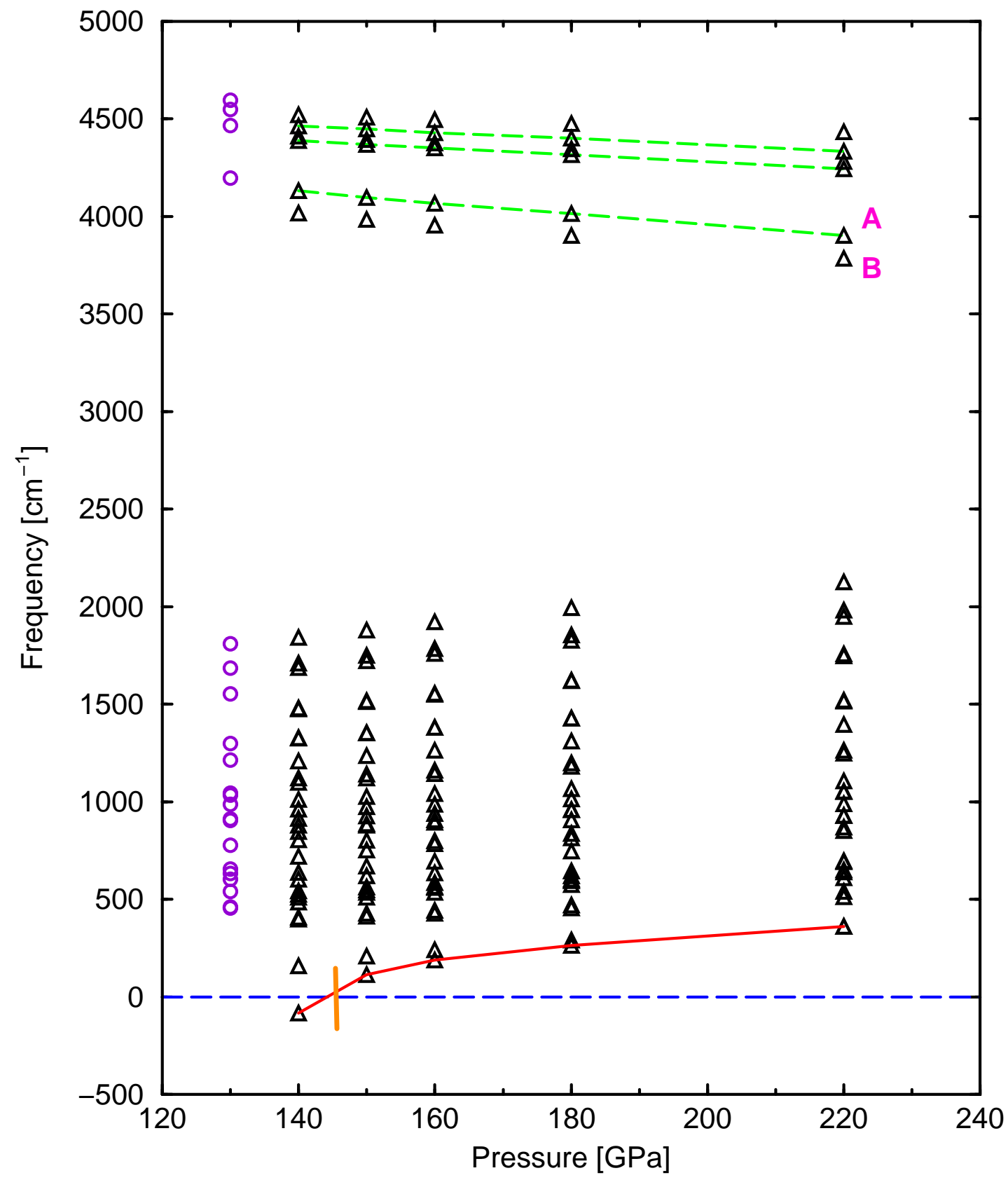




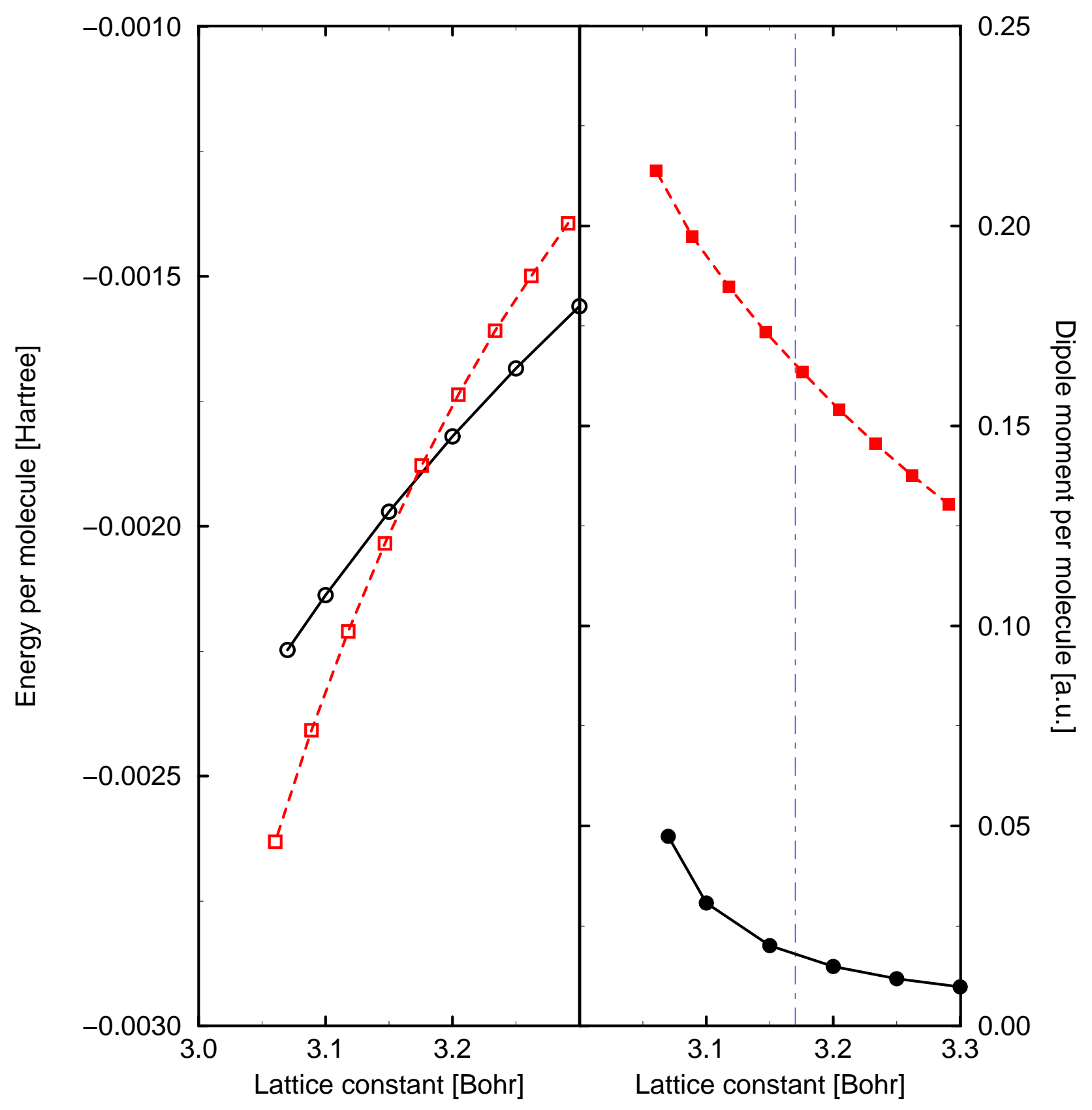

